\title{
Double-scale homogenized impedance models for periodically modulated metasurfaces
}

\author{
Enrica Martini ${ }^{1,}$, Francesco Caminita ${ }^{2}$, and Stefano Maci ${ }^{1}$ \\ ${ }^{1}$ Department of Information Engineering and Mathematics, University of Siena, Siena, Italy \\ ${ }^{2}$ Wave Up Srl, Siena, Italy
}

Received: 1 November 2020 / Accepted: 11 December 2020

\begin{abstract}
This paper investigates the accuracy of homogenized impedance models for the description of periodically modulated metasurfaces (MTSs) realized by printing subwavelength patches on a grounded dielectric slab. The problem is relevant to surface-wave based MTS antennas. The homogenized models are based on the local impedance synthesis of the subwavelength patch elements on the basis of a micro-periodicity assumption (that is, with a subwavelength period); the homogenized impedance is successively used in a macroperiodically modulated problem; that is, a periodic homogenized problem with a period which includes several micro-periods. Two different homogenized impedance models are compared. A first model is based on an anisotropic "impenetrable" impedance, defined by boundary conditions (BCs) at the MTS-air interface, while the second one uses a "penetrable" impedance sheet describing the homogenized BCs imposed by the metallic cladding on the grounded metallic slab. Although the presence of the grounded slab is considered in both models, they provide different results when the homogenized impedance is used to describe the macro-modulation. It is shown, through comparison with a full-wave analysis, that both the homogenized models can provide consistent results, but the penetrable impedance model is more accurate in the prediction of both the complex propagation constant and the current distribution. This is due to its capability to correctly account for the spatial dispersivity of the MTS.
\end{abstract}

Keywords: Metasurface / equivalent impedance model / leaky wave antennas / periodic structures

\section{Introduction}

Modulated metasurface (MTS) antennas are based on the interaction between the surface wave (SW) excited by a coplanar feeder and an MTS with a locally periodic modulation designed to convert the SW into a leaky wave $(\mathrm{LW})$ with prescribed characteristics $[1,2]$. The design is generally done starting from the definition of continuous boundary conditions (BCs) of (possibly anisotropic) impedance type, and then proceeding to its practical implementation. In the microwave range, this latter is typically performed through a distribution of electrically small patches printed over a grounded dielectric slab, and the modulation is achieved by gradually changing size, shape and/or orientation of the patches. In the resulting multiscale model, one can identify two geometrical scales, depicted in Figure 1. A local "microscale", determined by the subwavelength unit cell of the patch-element (denoted herein after as "microperiod") and a "macroscale", dictated by the period of the impedance modulation (denoted as "macroperiod"). The modulation, and therefore, the

\footnotetext{
* e-mail: martini@dii.unisi.it
}

definition of the macro-period may change in different zones of the MTS antenna, for the purpose of controlling the power density distribution [3] or the bandwidth $[4,5]$. The synthesis of the elements can be also performed at two different scale levels, corresponding to the two different periodic problems described above. At microscale, the synthesis is obtained by associating to each patch the impedance value obtained by analyzing that patch in a micro-periodic environment and by resorting to an equivalent transmission line model for the dominant Floquet mode [6]. Such an approach is obviously rigorous for non-modulated (uniform) MTSs, consisting of identical unit micro-cells. At macroscale, one can use the same conceptual approach, but generalized to the case of a macro-period. However, this second approach is computationally very time consuming, due to the large number of parameters needed for the construction of the full-wave data base. Therefore, a double-scale synthesis approach has been proposed in the past $[7,8]$ which makes use of both scales in the description of the homogenized impedance. This approach is based on two steps. In a first step, the micro-periodic problem is used for the identification of the patch geometrical parameters which realize a desired local impedance. In a second step, the macro-periodic problem is 


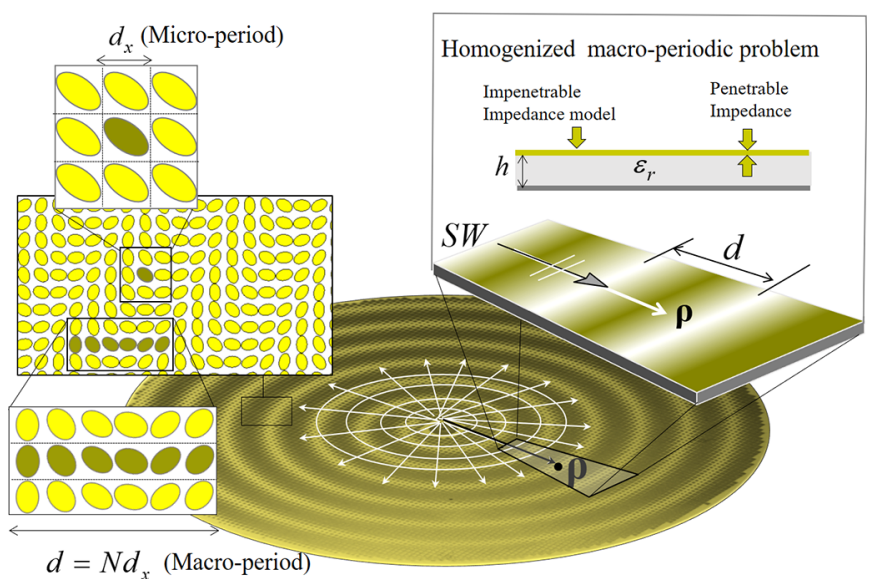

Fig. 1. MTS antenna and illustration of the double-scale periodicity (left hand zoom) and of the homogenized impedance models (rigth hand side zoom).

used with the homogenized impedance to identify the parameters for the antenna synthesis, namely the propagation constant, the leakage parameter and the field polarization.

The double-scale synthesis has already been proved very accurate for several different structures [6,9]; however, one might question its accuracy, especially in the presence of strong modulations, in which adjacent patches may exhibit quite different characteristics. The rigorous investigation of this accuracy is the main objective of this paper. This investigation is conducted through the analysis of the problem of an MTS with a 1D sinusoidal modulation, which represents the building block for the analysis of a large class of modulated MTS antennas. Although here we focus the attention on SW-to-LW coupling, a similar approach can be developed for MTS-based space wavecoupling devices based on locally periodic boundary conditions (BCs), such as deflectors or spatial filters. Also, two different homogenized impedance models are considered and compared here, namely, the one based on an impenetrable impedance, and the one based on a penetrable impedance sheet on a grounded slab describing the metallic cladding [6].

The analysis of the homogenized model is done by applying a generalization of the procedure proposed by Oliner and Hessel [10] for isotropic impenetrable impedance in their seminal paper. The generalization concerns the inclusion of anisotropy and the extension to penetrable BCs. Then, in order to test the capability of the homogenized models to predict the behavior of real MTSs, a rigorous full-wave analysis has been applied to analyze patch-based implementation of the same impedance profile. To this end, the periodic spectral Method of Moments (MoM) described in [11] has been applied to an entire modulation period.

The comparison among the different ways to extract the local homogenized impedance shows that both models can provide consistent results, but the one based on penetrable impedance offers much better accuracy in the

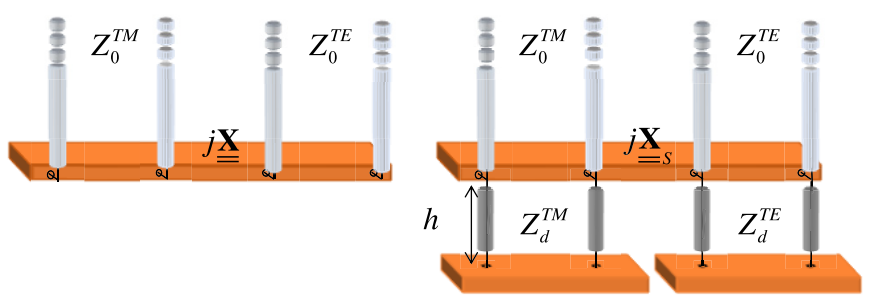

Fig. 2. Equivalent transmission line models for the IIBC (left) and the PIBC (right).

prediction of both the complex propagation constant and the vector current distribution. These quantities are all fundamental in the control of the aperture field distribution of MTS antennas in terms of phase, amplitude and polarization. The penetrable impedance model has been shown to also provide higher stability in numerical methods based on integral equations [12]. On the other hand, the opaque impedance model is less accurate, especially in the prediction of the attenuation constant. It is worth noting, however, that for different implementations of the MTS, like the one with fakir bed [13], the impenetrable impedance model is the only possibility; in the latter case, luckily, the weak dispersivity of the support is much less influent on the accuracy of the parameters extraction.

This work is organized as follows. Section 2 introduces the penetrable and impenetrable equivalent impedance models for a uniform MTS. Section 3 presents the generalization of the Oliner's procedure for the analysis of a sinusoidally modulated MTS, using an isotropic or anisotropic penetrable or impenetrable impedance model. Section 4 describes a rigorous full-wave approach for the analysis of a periodic MTS consisting of patches printed on a grounded slab. Section 5 presents numerical results. Finally, conclusions are drawn in Section 6 .

\section{Homogenized impedance models at microscale and macroscale}

\subsection{Microscale uniform MTSs}

We consider here MTSs consisting of electrically small patches printed on a grounded dielectric slab of relative permittivity $\varepsilon_{r}$ and thickness $h$ (see Fig. 1). The patches lie on the $x y$ plane of a Cartesian reference system. In the following, we neglect losses and assume purely reactive impedances. Although approximate, this model is able to capture the dominant phenomena in the microwave frequency range.

For a uniform MTS, consisting of identical unit cells centered at the nodes of a rectangular lattice, the periodicity allows for the rigorous definition of average tangential fields in a simple way, using a local Floquetwave (FW) expansion. Basically, the average electric and magnetic fields coincide with the corresponding coefficients of the dominant, zero-indexed FW. For this mode, an 
equivalent transmission line model can be defined, as shown in Figure 2. In this model, two different equivalent transmission lines are associated to TE (h) and TM (e) polarizations, for each of them the grounded slab is accounted for by a section of short circuited transmission line, while the metallic cladding is represented by a shunt load that, in the more general case, couples the TE and TM transmission lines.

Two different impedance BC models are then possible: the first one is referred to the impenetrable impedance $\mathrm{BC}$ (IIBC) at the patch level, and it relates the average tangential electric and magnetic average fields at $z=0^{+}$ through the impenetrable tensor impedance $j \underline{\underline{X}}$ as

$$
\mathbf{E}_{t}\left(z=0^{+}\right)=j \underline{\underline{X}} \hat{\boldsymbol{z}} \times \mathbf{H}_{t}\left(z=0^{+}\right) .
$$

This impenetrable equivalent impedance accounts for the contribution of both the patch cladding and the grounded slab in a unique impedance parameter $j \underline{\underline{X}}$.

The second model is referred to the penetrable impedance BC (PIBC), which can be written in terms of the discontinuity of the average tangential magnetic field across the metallization as follows

$$
\mathbf{E}_{t}(z=0)=j \underline{\underline{X}}_{S} \hat{\boldsymbol{z}} \times\left(\mathbf{H}_{t}\left(z=0^{+}\right)-\mathbf{H}_{t}\left(z=0^{-}\right)\right) .
$$

The penetrable tensor impedance $j \underline{\underline{X}}_{S}$ only represents the contribution of the metallic cladding.

The tensor impedances can be reduced to scalar quantities when the propagation direction is along a symmetry axis of the unit cell, that is, in absence of crosscoupling between the two polarizations.

Both the equivalent impedances defined above are in general dependent on wavenumber, besides on frequency. However, this dependency is much stronger for the impenetrable impedance, due to the contribution of the grounded slab. This is the main reason of the higher accuracy of the penetrable impedance model, which will be shown in the following.

\subsection{Macroscale modulated MTSs}

In patch-based MTSs, modulation is realized by gradually changing the size, shape or orientation of the patches in adjacent unit cells. At the analysis level, the corresponding homogenized impedance model is generally defined based on a local periodicity approximation. According to this approximation, the equivalent impedance value at each unit cell position is determined by analyzing the unit cell in a periodic environment (see Fig. 1).

Similarly, local periodicity approximation can be also used at the synthesis level, that is, for the practical patch implementation of a desired impedance profile [6]. In this case, different unit cells are studied in a periodic environment to construct a database relating the equivalent impedance to one or more geometrical parameters of the patches.

\section{Homogenized macroscale analysis}

We consider now the macroscale problem of determining the propagation characteristics and field distributions of

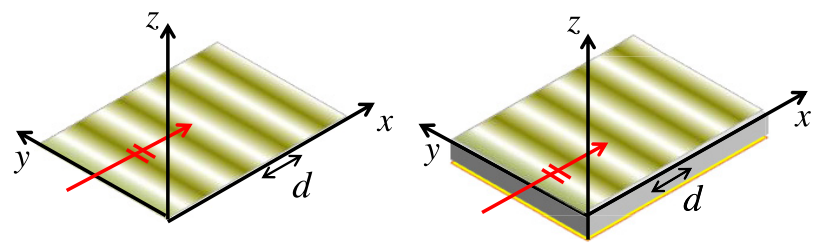

Fig. 3. Geometry for the problem of the sinusoidally modulated impedance. Left: impenetrable impedance. Right: penetrable impedance on a grounded slab.

waves supported by impedance type BCs modulated sinusoidally along the propagation direction. For an impenetrable scalar impedance, this problem has been addressed by Oliner and Hessel [10] with a rigorous numerical approach based on the FW expansion of modal currents and fields. More recently, that approach has been generalized to the case of tensor and/or penetrable impedance [14]. In this paragraph, we briefly review this generalized formulation.

The geometry for the problem is shown in Figure 3. A rectangular reference system is assumed with the $z$ axis orthogonal to the metasurface and the origin at the metasurface level. The MTS is modulated along $x$ and uniform along $y$. For this reason, in the following, we only explicitly indicate the dependency on $x$, implicitly assuming that all the quantities are evaluated at $z=0$.

With reference to this geometry, the TM (e) and TE (h) components of the electric current and electric field are along $x$ and $y$, respectively. The tensor impedance which relates the tangential electric field and current according to the relationship.

$$
\mathbf{E}(x)=\underline{\underline{\mathbf{Z}}}(x) \cdot \mathbf{I}(x)
$$

is written as

$\underline{\underline{\mathbf{Z}}}(x)$

$=\left[\begin{array}{cc}j \bar{X}_{e e}\left[1+m_{e e} \cos \left(\kappa x+\varphi_{e e}\right)\right] & j X_{e h} m_{e h} \cos \left(\kappa x+\varphi_{e h}\right) \\ j X_{e h} m_{e h} \cos \left(\kappa x+\varphi_{e h}\right) & j \bar{X}_{h h}\left[1+\mathrm{m}_{h h} \cos \left(\kappa x+\varphi_{h h}\right)\right]\end{array}\right]$

with $\kappa=\frac{2 \pi}{d}$, where $d$ is the modulation period, $\mathbf{I}=\left[\begin{array}{l}I_{e} \\ I_{h}\end{array}\right]=\left[\begin{array}{l}I_{x} \\ I_{y}\end{array}\right]$ and $\mathbf{E}=\left[\begin{array}{c}E_{e} \\ E_{h}\end{array}\right]=\left[\begin{array}{c}E_{x} \\ E_{y}\end{array}\right] . \operatorname{In}(3), \mathbf{I}=$ $\hat{\boldsymbol{z}} \times \mathbf{H}_{t}$ and $\underline{\underline{\mathbf{Z}}}=j \underline{\underline{\mathbf{X}}}$ for IIBC, while $\mathbf{I}=\hat{\boldsymbol{z}} \times\left(\mathbf{H}_{t}^{+}-\mathbf{H}_{t}^{-}\right)$ (current flowing in the metallic cladding) and $\underline{\underline{\mathbf{Z}}}=j \underline{\underline{\mathbf{X}}}$ for PIBC

Due to the periodicity of the problem, we can expand the electric current density in Floquet Wave (FW) series

$$
I_{e, h}=\sum_{n} I_{e, h}^{(n)} e^{-j k_{x n} x}
$$

where $I_{e, h}^{(n)}$ denotes the coefficient of the $n$-th FW of the TM, TE current component, $k_{x n}=k_{x 0}+n \frac{2 \pi}{d}$ and $k_{x 0}$ represents the propagation constant of the 0 -indexed $\mathrm{FW}$, from 
which all the other FW wavenumbers are immediately obtained.

The electric field can be also expanded in FW series

$$
E_{e, h}=\sum_{n} E_{e, h}^{(n)} e^{-j k_{x n} x}
$$

where

$$
E_{e, h}^{(n)}=\int_{0}^{d} E_{e, h} e^{j k_{x n} x} d x
$$

After expressing the cosine functions in the impedance tensor in terms of complex exponentials and combining (3) with (7) we get

\section{See equation (8) below.}

For each FW of this expansion, the electric field is related to the current by the spectral Green's function (GF) of the problem, evaluated at the corresponding wavenumber:

$$
E_{e, h}^{(n)}=\tilde{G}_{E J}^{e, h}\left(k_{x n}, 0\right) I_{e, h}^{(n)} e^{-j k_{x n} x}
$$

where $\tilde{G}_{E J}^{e, h}\left(k_{x}, k_{y}\right)$ is the spectral TM,TE Green's function relating the electric current to the electric field. The GF $\tilde{G}_{E J}^{e, h}$ is defined in a different way according to the type of $\mathrm{BC}$ model adopted. For IIBC, the GF is the one of the free space (Fig. 2, left hand side), for PIBC the GF accounts for both free space and the grounded slab (Fig. 2, right hand side).

After inserting equation (9) into (8) and collecting the equations for TM and TE components, we obtain the following homogenous system of equations:

$$
\underline{\underline{M}}_{P} \mathbf{I}_{n+1}+\underline{\underline{D}}_{n} \mathbf{I}_{n}+\underline{\underline{M}}_{M} \mathbf{I}_{n-1}=0
$$

where

$$
\underline{\underline{M}}_{P}=-\left(\underline{\underline{M}}_{M}\right)^{*}=\left[\begin{array}{cc}
j \frac{\bar{X}_{e e} m_{e e} e^{j \psi_{e e}}}{2} & j \frac{X_{e h} m_{e h} e^{j \psi_{e h}}}{2} \\
j \frac{X_{e h} m_{e h} e^{j \psi_{e h}}}{2} & j \frac{\bar{X}_{h h} m_{h h} e^{j \psi_{h h}}}{2}
\end{array}\right]
$$

and

$$
\underline{\underline{D}}_{n}=\left[\begin{array}{cc}
j X_{e e}-\tilde{G}_{E J}^{e}\left(k_{x n}\right) & 0 \\
0 & j X_{h h}-\tilde{G}_{E J}^{h}\left(k_{x n}\right)
\end{array}\right] .
$$

Equation (11) can be viewed as a double infinite set of linear homogenous equations with an infinite number of vector unknowns $\mathbf{I}_{n}=\left[\begin{array}{c}I_{e}^{(n)} \\ I_{h}^{(n)}\end{array}\right]$, whose solutions represent the current modes supported by the structure. In practice, the system can be truncated by considering a finite number of FWs introducing a negligible error. The resulting set of equations possesses a nontrivial solution only if the determinant of the linear system vanishes. This condition is solved with respect to the variable $k_{x 0}$, the propagation constant of the 0 -indexed FW.This is the reason why it is fundamental, in order to get an accurate solution, to correctly account for the dependency of the system coefficients on the propagation constant. For the value of $k_{x 0}$ which nullifies the determinant, the system matrix has a null eigenvalue; the eigenvector corresponding to this null eigenvalue contains the coefficients of the FW modal expansion of the modal current which propagates with propagation constant $k_{x 0}$.

This propagation constant can be real or complex, corresponding to $\mathrm{SWs}$ or $\mathrm{LWs}$, respectively. For LW modes, the real part of the propagation constant of one of the FWs falls inside the visible region; accordingly, the imaginary part of the propagation constant accounts for progressive power leakage due to radiation. The solution of the dispersion equation is usually expressed in the form $k_{x 0}=\beta_{0}+\Delta \beta-j \alpha$, where $\beta_{0}$ is the propagation constant of the unmodulated impedance surface, in order to immediately identify the perturbation induced by the modulation.

Tailoring the characteristics of LWs is the key step for the design of MTS antennas. In fact, in these structures the real part and imaginary part of the propagation constant control the phase and the amplitude of the aperture field, respectively, while the vector current of the radiating mode controls the polarization.

\section{Macro-scale exact full wave analysis of periodically modulated patch-based MTS}

A rigorous analysis of the periodically modulated patchtype MTS can be obtained by analyzing with the Method of Moments (MoM) the single modulation period with

$$
\begin{aligned}
E_{e}^{(n)}= & j \bar{X}_{e e} I_{e}^{(n)}+\frac{1}{2} j \bar{X}_{e e} m_{e e} \mathrm{e}^{-j \varphi_{e e}} I_{e}^{(n+1)}+\frac{1}{2} j \bar{X}_{e e} m_{e e} \mathrm{e}^{j \varphi_{e e}} I_{e}^{(n-1)} \\
& +j X_{e h} m_{e h} \mathrm{e}^{-j \varphi_{e h}} I_{h}^{(n+1)}+j X_{e h} m_{e h} \mathrm{e}^{j \varphi_{e h}} I_{h}^{(n-1)} \\
E_{h}^{(n)}= & j \bar{X}_{h h} I_{h}^{(n)}+\frac{1}{2} j \bar{X}_{h h} m_{h h} \mathrm{e}^{-j \varphi_{h h}} I_{h}^{(n+1)}+\frac{1}{2} j \bar{X}_{h h} m_{h h} \mathrm{e}^{j \varphi_{h h}} I_{h}^{(n-1)} \\
& +j X_{e h} m_{e h} \mathrm{e}^{-j \varphi_{e h}} I_{e}^{(n+1)}+j X_{e h} m_{e h} \mathrm{e}^{j \varphi_{e h}} I_{e}^{(n-1)}
\end{aligned}
$$


periodicity BCs. A spectral MoM approach like the one described in [11] is used in this paper.

The geometry of the problem is shown in Figure 4. The analysis domain has dimensions $N d_{x}=d$ along $x$, where $N$ is the number of patches for modulation period, and $d_{y}$ along $y$, respectively. Fields and currents are expanded in series of FWs with wave vectors located at the "reciprocal lattice" nodes $\mathbf{k}_{q}$. Notice that in this case the periodicity is $2 \mathrm{D}$, even if we restrict our analysis to wave propagating along the $x$ direction, so that the generic $\mathrm{FW}$ wave vector will be $\mathbf{k}_{q}=\mathbf{k}_{n m}=\left(k_{x 0}+n \frac{2 \pi}{N d_{x}}\right) \hat{x}+m \frac{2 \pi}{d_{y}} \hat{y}$. After using a proper set of basis functions to expand the currents in each patch and applying a Galerkin spectral approach and the Poisson summation formula, the MoM mutual impedance takes on the form [11]:

$$
Z_{l j}^{M o M}=\sum_{q=0}^{M-1} \tilde{\mathbf{J}}_{l}^{*}\left(\mathbf{k}_{q}\right) \cdot\left[\tilde{G}_{E J}^{e}\left(\mathbf{k}_{q}\right) \hat{\sigma}_{q} \hat{\sigma}_{q}+\tilde{G}_{E J}^{h}\left(\mathbf{k}_{q}\right) \hat{\alpha}_{q} \hat{\alpha}_{q}\right] \cdot \tilde{\mathbf{J}}_{j}\left(\mathbf{k}_{q}\right)
$$

where $\tilde{\mathbf{J}}_{l}\left(\mathbf{k}_{q}\right)\left[\tilde{\mathbf{J}}_{j}\left(\mathbf{k}_{q}\right)\right]$ is the Fourier transform of the basis [test] function $\mathbf{j}_{l}(\boldsymbol{\rho})\left[\mathbf{j}_{j}(\boldsymbol{\rho})\right]$, sampled at the $\mathrm{FW}$ wave vectors $\mathbf{k}_{q}, \hat{\sigma}_{q}=\mathbf{k}_{q} / \sqrt{\mathbf{k}_{q} \cdot \mathbf{k}_{q}}, \hat{\alpha}_{q}=\hat{z} \times \hat{\sigma}_{q}$, and the asterisk denotes conjugation.

Once the MoM impedance matrix is known, the propagation constant of the supported mode is obtained by setting to zero its determinant.

$$
\operatorname{det}\left[\mathbf{Z}^{M o M}\left(k_{x 0}\right)\right]=0 .
$$

In the following, we consider circular or elliptical patches, for which entire domain basis functions can be defined, which allow for an extremely accurate and efficient analysis [15]. Only two basis functions per patch have been demonstrated there to be sufficient to obtain very accurate results. Hence, the matrix in equation (14) has size $2 N \times 2 N$. Furthermore, these basis functions have a closed-form spectrum, which can be directly used in (13), with no need of any numerical integration for the calculation of the Fourier transform.

\section{Numerical results}

In this section we present some numerical results obtained by considering a dielectric slab characterized by a relative permittivity $\varepsilon_{r}=9.8$ and a thickness $h=0.508 \mathrm{~mm}$. The micro-period unit cell is square with size $1.25 \mathrm{~mm}$. Analyses are conducted at $26 \mathrm{GHz}$.

\subsection{Isotropic MTS}

The first analyses have been conducted on MTSs consisting of circular patches. In this case, the equivalent impedance tensor is diagonal $\left(X_{e h}=0\right.$ in Eq. (4)), and the supported modes are purely TM. The modulation macro-period consists of 8 patches, corresponding to an overall length of $1 \mathrm{~cm}$.

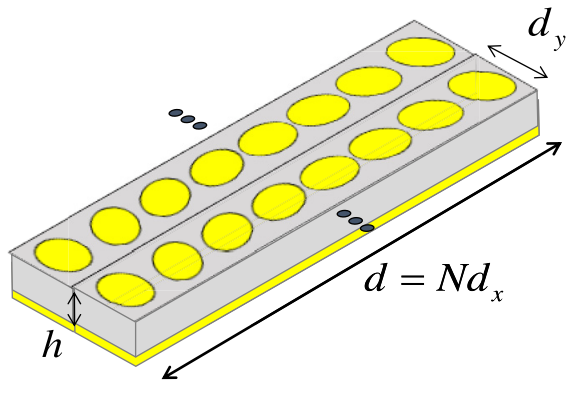

Fig. 4. Geometry for the periodically modulated patch-type MTS.

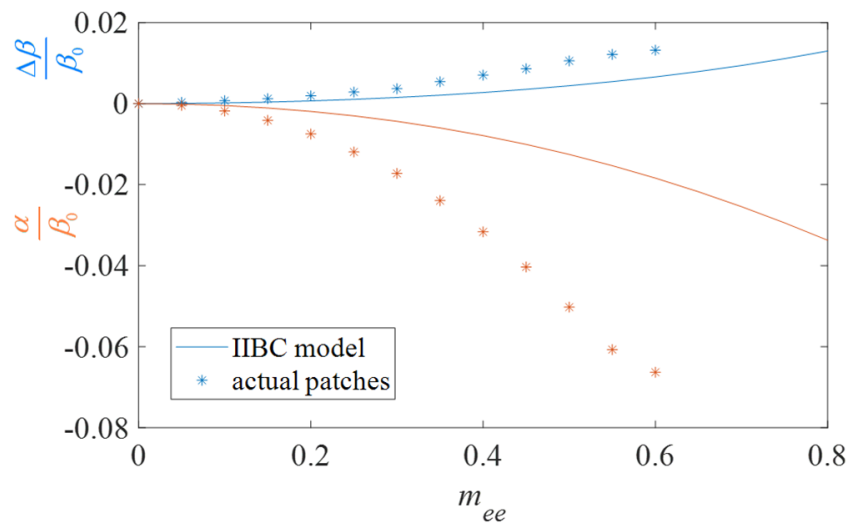

Fig. 5. Normalized real and imaginary part of the perturbation of the propagation constant on a modulated MTS. Comparison between IIBC model and rigorous full wave analysis for different values of the modulation index.

Both IIBC and PIBC models are considered. In both the cases, a continuous impedance profile with a sinusoidal modulation has been first defined, and then implemented through patches of variable size. As explained in Section 2.2, this synthesis step is based on maps generated invoking the local micro-periodicity approximation. The results provided by the homogenized models described in Section 3 are compared with the ones provided by the rigorous full-wave analysis described in Section 4 .

For the IIBC case, we have chosen $\bar{X}_{e e}=0.8 \zeta_{0}$, where $\zeta_{0}$ is the free space impedance and we have varied the modulation index $m_{e e}$ in the range [0 0.6$]$. The average impedance corresponds to a propagation constant $\beta_{0}=$ $698 \mathrm{~m}^{-1}$. Numerical results obtained for the propagation constant are shown in Figure 5.

The results provided by the IIBC model are quite accurate for the real part of the propagation constant (notice that the plot reports the normalized displacement from the value corresponding to the average impedance, the percent error on the real part is smaller than 1\%), but they are less accurate in the prediction of the attenuation constant. This is due to the dispersive nature of the impenetrable impedance, not accounted for in the model.

A similar analysis has been conducted for the PIBC model. Notice that it was not possible to analyze the same problem with the two models, due to the assumption of 


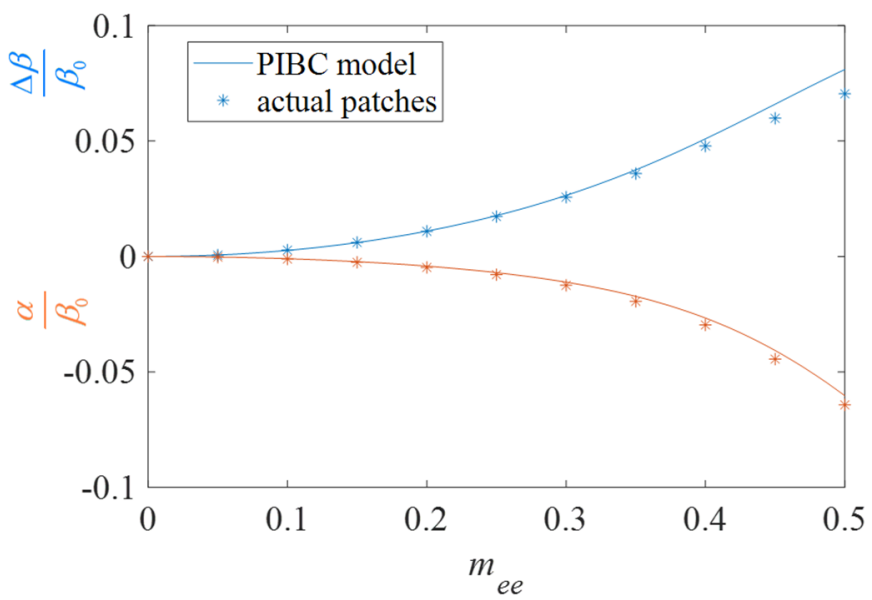

Fig. 6. Normalized real and imaginary part of the perturbation of the propagation constant on a modulated MTS. Comparison between penetrable impedance model and rigorous full wave analysis for different values of the modulation index.

sinusoidal modulation. In fact, a sinusoidally modulated IIBC does not correspond to a sinusoidally modulated PIBC. For the PIBC we have chosen an average value $\bar{X}_{e e}=-200 \Omega$, corresponding to $\beta_{0}=665 \mathrm{~m}^{-1}$, similar to the one of the IIBC case, and we have varied the modulation index $m_{e e}$ in the range [0 0.5], so as to obtain attenuation constants of the same order of magnitude of the ones shown in Figure 5. Numerical results are shown in Figure 6. It can be seen that the PIBC model results are very accurate for both the propagation and the attenuation constant, even for strong modulations, corresponding to faster variations in the geometry of the patches. The maximum percent error on the real and imaginary part of the propagation constant are less than $0.002 \%$ and around $6 \%$, respectively.

For the PIBC model, we have also looked at the current distribution. While the homogenized model provides a continuous current profile, from the MoM method we just have a basis function coefficient for each patch. In fact, in this isotropic case, the supported mode is purely transverse magnetic, and only one basis function for patch is sufficient to accurately describe the current. As shown in Figure 7 for the case $m_{e e}=0.4$, the coefficients provided by the MoM method sample the continuous curve predicted by the PIBC with a remarkably good agreement. The same figure also shows the current on the patches as predicted by MoM.

It is noted that the calculation based on the PIBC model is practically instantaneous (less than $1 \mathrm{~s}$ for the whole curve on an ordinary personal computer), while the full wave analysis requires appriximately $20 \mathrm{~s}$ for each point. In this latter case, computation is significantly speeded up by the use of the entire domain basis functions in [15], ant it would require much more time if Rao-WiltonGlisson (RWG) [16] basis functions were used.

\subsection{Anisotropic MTS}

Finally, we have also considered an anisotropic modulated MTS and we have evaluated the capability of the

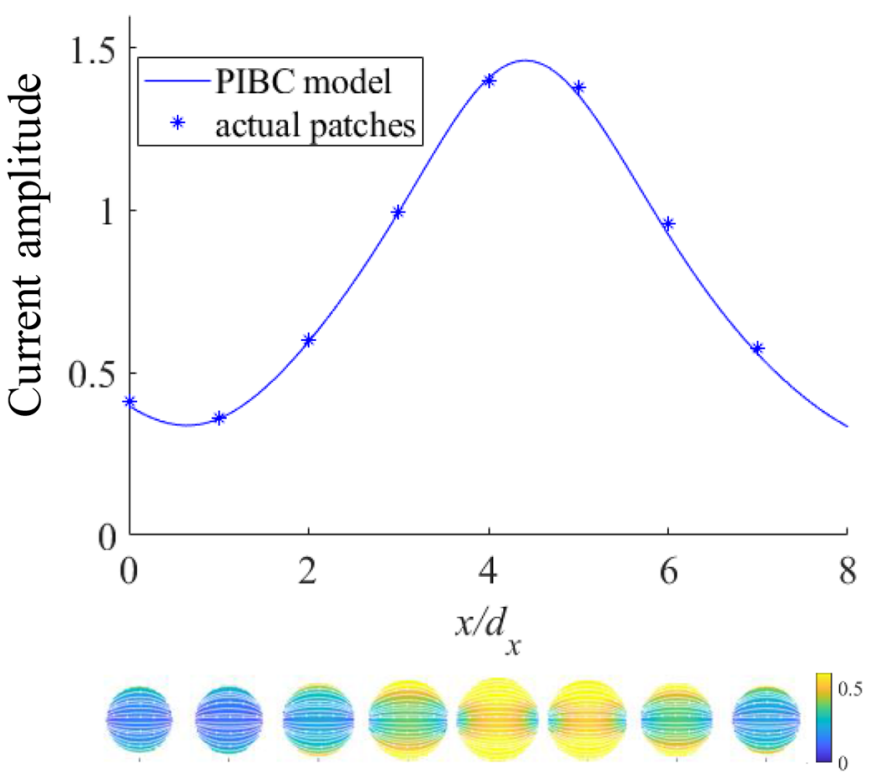

Fig. 7. Current amplitude on a modulated MTS: comparison between PIBC model and MoM. The color plot in the bottom shows the current on patches according to MoM solution.

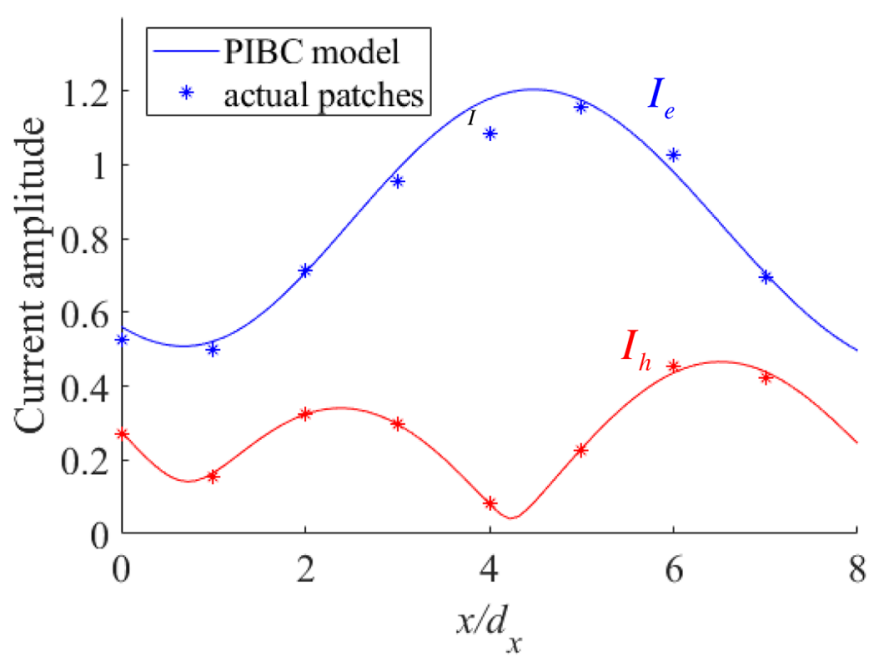

Fig. 8. Current amplitude on a modulated MTS: comparison between PIBC model and MoM.

homogenized impedance model to correctly predict the vector current across the MTS. To this end, the eigenvectors corresponding to the null eigen value have been obtained both for the analysis based on PIBC and from the one based on spectral MoM. The results for the modulation parameters $m_{e e}=m_{e h}=m_{h h}=0.25$, $\bar{X}_{e e}=X_{e h}=\bar{X}_{h h}=-200 \Omega, \psi_{e e}=\psi_{h h}=0, \psi_{e h}=\pi / 2$, are compared in Figures 8 and 9. In Figure 8, the amplitudes of the TE and TM components of the current are shown. As for the isotropic case, the discrete values obtained for the patch-based MTS are very close to the continuous curve provided by the homogenized model.

In Figure 9, the currents from the PIBC model have been sampled in correspondence of the patch centers and 


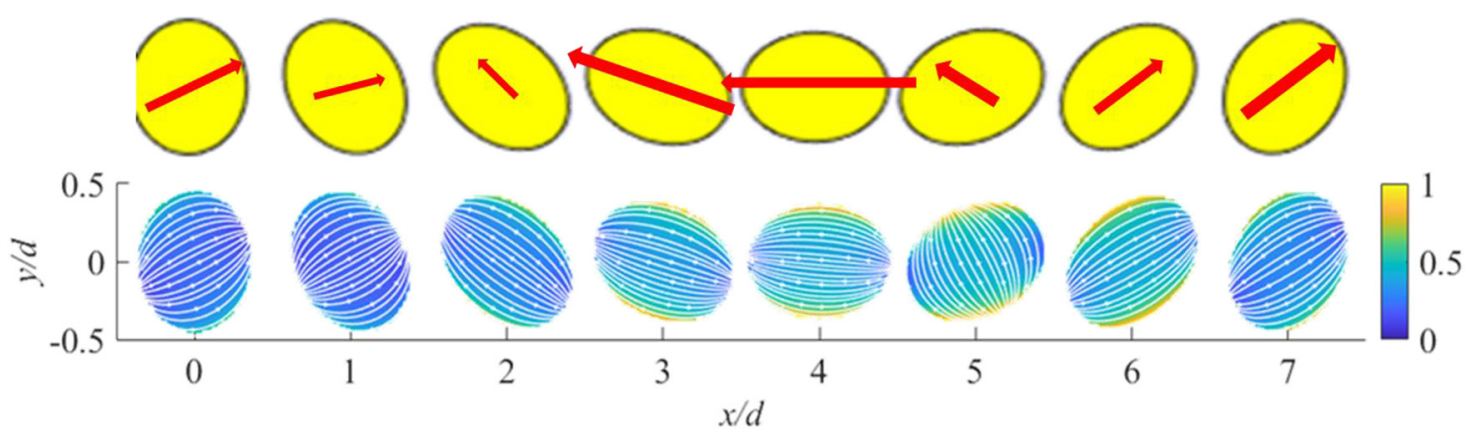

Fig. 9. Real part of the vector currents across a period of a modulated MTS. Top line: currents provided by the PIBC model sampled at the center of the single unit cells. Bottom line: currents on patches provided by the spectral MoM with entire domain basis functions.

compared with the corresponding MoM results. As it can be seen, there is a remarkable agreement between the two representations both in the amplitude (represented by the length of the arrow in the first plot and by the color in the second plot) and in the orientation (direction of the arrows) of the currents. In particular, it is interesting to observe the current on the first patch. This patch has its axes aligned with the $x$ and $y$ axes, which means that the wave is propagating along one of its principal axes. This implies that in a strictly periodic environment the current would be aligned along $x$. However, as shown by the results of the spectral MoM analysis, this is not the case, due to the presence of rotated patches in the adjacent unit cells. Quite remarkably, the PIBC model is able to correctly predict this effect, which cannot be predicted by the analysis of the single unit cell.

\section{Conclusion}

The accuracy of penetrable and impenetrable double-scale homogenized impedance models of periodically modulated MTS has been investigated. The investigation was conducted by comparing the results obtained with the homogenized models with the ones provided by a MoM analysis which rigorously takes into account the dielectric stratification (through the Green's function) and the exact shape of the patches (through the basis functions). It is shown that both the models can accurately predict the propagation constant of the supported LW, but the accuracy in the prediction of the attenuation constant is significantly better for the PIBC model, thanks to the reduced spatial dispersion in describing the impedance parameters.

Also, the PIBC model is able to correctly predict the current behavior across the periodic MTS. This analysisvalidates both the PIBC model and the double-scale approach used to extract the equivalent impedance. In fact, even if the equivalent impedance is extracted by resorting to a local periodicity assumption, the model based on homogenized penetrable impedance is able to correctly describe the current and field behavior in terms of both amplitude and polarization in the modulated macroperiodic environment, which can be significantly different from the one in the micro-periodic environment. The PIBC double-scale homogenization model provides therefore a powerful tool for the analysis and design of MTS antennas.

\section{References}

1. B.H. Fong, J.S. Colburn, J.J. Ottusch, J.L. Visher, D.F. Sievenpiper, IEEE Trans. Antennas Propag. 58, 3212 (2010)

2. M. Faenzi, G. Minatti, D. González-Ovejero, F. Caminita, E. Martini, C. Della Giovampaola, S. Maci, Sci. Rep. 9, 10178 (2019)

3. G. Minatti, E. Martini, S. Maci, IEEE Trans. Antennas Propag. 65, 1532 (2017)

4. G. Minatti, M. Faenzi, M. Sabbadini, S. Maci, IEEE Trans. Antennas Propag. 65, 2836 (2017)

5. M. Faenzi, D. González-Ovejero, S. Maci, IEEE Trans. Antennas Propag. 68, 1261 (2020)

6. E. Martini, M.jr Mencagli, S. Maci, Philos. Trans. Royal Soc. A 373, 20140355 (2015)

7. G. Minatti, F. Caminita, E. Martini, S. Maci, IEEE Trans. Antennas Propag. 64, 3896 (2016)

8. G. Minatti, F. Caminita, E. Martini, M. Sabbadini, S. Maci, IEEE Trans. Antennas Propag. 64, 3907 (2016)

9. D. González-Ovejero, S. Maci, IEEE Trans. Antennas Propag. 63, 3982 (2015)

10. A. Oliner, A. Hessel, IRE Trans. Antennas Propag. 7, $201(1959)$

11. S. Maci, A. Cucini, FSS-based EBG surfaces, in Electromagnetic Metamaterials: Physics and Engineering Aspects, edited by N. Engheta, R. Ziolkowski (Wiley, Hoboken, NJ, 2006), pp. $351-376$

12. M.A. Francavilla, E. Martini, S. Maci, G. Vecchi, IEEE Trans. Antennas Propag. 63, 2153 (2015) 
13. M.G. Silveirinha, C.A. Fernandes, J.R. Costa, IEEE Trans. Antennas Propag. 56, 405 (2008)

14. F. Caminita, E. Martini, S. Maci, in 2015 9th European Conference on Antennas and Propagation (EuCAP), Lisbon (2015)

Cite this article as: Enrica Martini, Francesco Caminita, Stefano Maci, Double-scale homogenized impedance models for periodically modulated metasurfaces, EPJ Appl. Metamat. 7, 12 (2020)
15. M. Mencagli, E. Martini, S. Maci, IEEE Trans. Antennas Propag. 63, 2992 (2015)

16. S.M. Rao, D.R. Wilton, A.W. Glisson, Electromagnetic scattering by surfaces of arbitrary shape, IEEE. Trans. Antennas Propag. AP-30, 409 (1982) 